can also improve communication with primary care services by providing GPs with a concise and succinct summary of the patients' problems and care plans,

special articles including relapse and risk information. We intend working with our GPs to establish eCPA care plan registers on their computer networks, once issues of data security have been addressed.

\section{Automation of form completion}

It may be found in the future that greater utility can be provided by including a greater level of automation into the forms. Already staff use 'Autotext' to store commonly used words and sentences. Future developments could include drop down menus so that the diagnosis or the drug dosage can be chosen from an on-screen listing, instead of having to be looked up and then typed in full. The form could be programmed with predictive text entry. It may be possible to adapt the form to link to a relational database to allow data to be drawn from or exported to patient or case records.

However, most of these advances would probably involve bespoke programming and would be difficult to implement while the eCPA form remains in its current format as a standard Microsoft Word template. For the present, the advantage of almost universal compatibility when e-mailing the documents probably outweighs the advantages that these innovations might offer.

\section{Conclusion}

The simple act of placing the paper-based CPA care plan form into an electronic format brings considerable benefits to CMHT staff and patients, providing an inexpensive, rapid method of improving the quality and communication of the care plans. Furthermore, these eCPA care plans provide an ideal medium for the dissemination and adoption of good practice by CMHT staff. Practice notes and form-based working will never replace the skill and experience of the team, but can provide a helpful tool to promote good practice - and reduce the potential for errors and omissions.

\section{Acknowledgements}

We wish to thank the staff of St Giles Team 3 and Ward ES2 for their support with this project, as well as Professor E. Kuipers and Mr Patrick Phokeerdoss, among others, for the provision of good practice points for conversion into Practips.

\section{Declaration of interest}

None.

\section{References}

APPLEBY, L. (2000) Safer services: conclusion from the report of the National Confidential Inquiry. Advances in Psychiatric Treatment, 6 $5-15$.

DEPARTMENT OF HEALTH (1999a) Effective Care Co-ordination in Mental Health Services: Modernising the Care ProgrammeApproach. London: Department of Health.

- (1999b) Mental Health, National Services Framework, Modern Standards and Service Models. London: Department of Health.

— (2000) Data Protection Act 1998 Protection and Use of Patient Information. Health Service Circular,
HSC 2000/009. London: Stationery Office. (http://www.doh.gov.uk/ pdfs/009hsc.pdf)

KEMP, R., HAYWARD, P. APPLEWHAITE, G., et al (1996) Compliance therapy in psychotic patients. BMJ, 312, 345-349.

PALMER, C. \& LELLIOTT, P. (2000) Encouraging the implementation of clinical standards into practice. Psychiatric Bulletin, 24, 90-93.

THORNICROFT, G. (2000) National Service Framework for Mental Health Psychiatric Bulletin, 24, 203-206.

WORLDHEALTH ORGANIZATION (1992) The ICD-10 Classification of Mental and Behavioural Disorders. Geneva: WHO

*Roger Howells Consultant Psychiatrist, AmandaThompsell Specialist Registrar, Maudsley Hospital, South London and MaudsleyTrust, Denmark Hill, London SE5 8AZ

\title{
Service innovations: development of a psychoeducational programme for patients with personality disorder
}

\section{AIMS AND METHOD}

This study describes a survey of in-patients with personality disorder admitted to a specialist personality disorder unit, in terms of their knowledge and understanding of their diagnosis, and the subsequent development of a psychoeducational programme.
RESULTS

Overall, patients had a poor knowledge and understanding of their diagnosis. Most had not had their diagnosis explained to them, and if they had, they had not retained the information. Consequently a structured psychoeducational programme was developed, incorporating diagnostic information obtained at the pre-admission assessment.

\section{CLINICAL IMPLICATIONS}

Diagnostic information for those with personality disorder, even when it is available, is not transmitted to patients. Imparting this information through a psychoeducational programme is one way in which the therapeutic relationship between patient and clinician can be strengthened. 
The Patient's Charter (NHS Executive, 1997) states that patients can expect to '. . . ask for an explanation of your diagnosis in plain language and to ask for more information if you do not understand'. Such an understanding is clearly a prerequisite to consent to treatment, the nature, purpose and likely effects of which depend on a knowledge of the diagnosis. But how much do patients with mental disorder really know about their diagnosis and does the type of disorder that they suffer from influence this understanding? In a survey of psychiatric out-patient and day hospital attendees, Stephen (2000) found that those patients with a primary diagnosis of personality disorder knew considerably less about their diagnosis than those with schizophrenia, bipolar disorder or depressive disorder. This finding is not surprising, given that the views of mental health professionals on both the validity and treatability of personality disorder are, themselves, sharply polarised (Lewis \& Appleby, 1988).

The personality disorder unit (PDU) at Arnold Lodge is a dedicated in-patient facility for the treatment of men with personality disorder with an offending history. Because one of the admission criteria is the presence of one or more personality disorder(s), referrals are assessed prior to admission with several self-report and interview measures to assess personality disorder. Consequently, there is no shortage of information on the personality of those who are admitted. This report documents a survey of the in-patients' understanding of their personality disorders and the development of a psychoeducational approach that utilised this assessment information as part of the therapeutic process.

\section{Patients' knowledge of their personality disorder diagnoses}

\section{Method}

All the patients resident on the PDU in January 2001 and who had been on the unit for at least a month $(n=11)$ were requested to complete a questionnaire, which asked three questions: (a) What is meant by the term personality disorder? (b) Are you aware of the specific type(s) of personality disorder(s) you have been assessed as having? (c) Has anyone discussed or explained your personality disorder diagnosis to you since you have been on the unit? Their responses to each question were rated by K.D. as 0 (no response), 1 (moderate response) or 2 (good response)

\section{Results}

With regard to their understanding of the term personality disorder, four (36\%) were rated as having no or a poor understanding, six (55\%) had a moderate understanding and only one (9\%) had a good understanding. With respect to knowledge of their specific personality diagnosis, two patients (18\%) knew their diagnostic categories (i.e. had good knowledge), two (18\%) knew of one category but not all (had some knowledge) and over half (seven patients, 64\%) had no knowledge at all. Of the four patients who had at least some awareness of their diagnostic categories, only one had been informed by a clinician and the other three had obtained this information indirectly from their care plan or reports. With regard to explanation or discussion of diagnosis, one patient (9\%) had it explained to him at length but had forgotten the explanation and two patients (18\%) had some explanation given by clinicians but again one of them had forgotten the information. The majority (eight patients, $73 \%$ ) had not had their diagnoses explained to them.

Given that this is a specialised personality unit and that the treatment of personality requires a collaborative approach if it is to be successful, these results were a cause for concern. This was especially the case because there was clearly a considerable amount of information in the patients' files; it appeared, however, that this was being denied to them. Consequently, one of us (K.D.) decided to develop a psychoeducational package that used the information that had been collected during preadmission to increase the individuals' awareness of our assessment of their disorders.

\section{A psychoeducational approach}

\section{Objectives and structure of the programme}

The primary objectives of the programme were to establish patients' knowledge of personality disorder in general, together with an understanding of the specific personality disorder(s) that the clinicians ascribed on the basis of the pre-admission assessment. A secondary objective was to enable the clinicians to review the evidence for diagnosis and to discuss with the patients whether or not the evidence justified this particular designation and to revise the pre-admission assessment where necessary. This might occur, for instance, where new information became available from collateral sources or from behavioural observations on the unit.

Although all patients referred to the PDU are assessed with a wide battery of personality disorder instruments pre-admission, special consideration is given to the findings from the interview version of the International Personality Disorder Examination (IPDE; Loranger et al, 1991). It has impressive reliability - at least by those who have been properly trained (Zimmerman, 1994) and it produces personality disorder diagnosis for both the DSM-IV (American Psychiatric Association, 1994) and ICD-10 (World Health Organization, 1992) systems of classification. It is a semi-structured examination that involves the interviewee being asked probe questions regarding the presence of the personality trait, and this is rated as being positive only if the interviewer can be convinced by a sufficient number of examples that the trait is pathological, pervasive and persistent. This instrument is especially useful for this exercise because each personality disorder is only rated as definite if a sufficient number of personality traits are rated as being positive. Consequently, a review of the assessor's original rating easily identifies the positive traits that have been rated 
and the subsequent revision commenced by reviewing these with the patient. The structure of the programme followed a definite pattern of regular weekly sessions with K.D. for 1 hour, lasting from 8 to 12 weeks (depending on the severity of the individual patient's psychopathology). The process of the programme is detailed below.

\section{Week 1}

Obtaining the patient's baseline knowledge of his diagnosis, asking him to identify his problems and how these may be due to his personality disorder and what interventions or treatment he thinks might help.

\section{Week 2}

Providing information on the concepts of personality and personality disorder in general and how the latter can give rise to difficulties.

\section{Week 3}

Provision of information on specific sub-categories of personality disorder, with reference to personality disorder clusters that relate to that individual.

\section{Week 4}

Encouraging and helping the patient to identify his own personality disorder diagnosis by asking him to go through a checklist of personality traits (derived from the IPDE scoring sheet) and identify those that apply to him.

\section{Weeks 5/6}

Comparing the patient's opinion with the findings obtained at the initial pre-admission IPDE assessment and also with that of his named nurse (who also was given an IPDE checklist to complete). In most cases the opinion of the patient, the named nurse and the findings at the preadmission assessment were in agreement. However, in cases where the patient was either unaware or tended to play down his psychopathology, this process was found to be particularly useful as a 'way in' to challenging the patient's perception of himself and his relationship with others. This step may take several sessions, depending on the severity of the individual's psychopathology.

\section{Week 7}

Making an attempt to understand the origin of the patient's disorder, considering both nature and nurture.

\section{Week 8}

Providing the patient with individualised written feedback in the form of a patient information booklet. This was considered to be especially important because our survey identified that, on the few occasions when verbal information was given to patients, it was frequently forgotten.

\section{Acceptability of the programme}

This programme was trialed on patients who had been admitted recently to the unit. On the whole, it was well received, with active engagement. The patients were particularly appreciative of the patient information booklet given at the end of the sessions. The main difficulty arose with patients with a diffuse disorder of personality who fulfilled the criteria for several personality diagnoses. Discussion of each of the traits characterising the disorder lengthened the programme and at times it was difficult to keep the patients' attention (and that of K.D.) focused.

This programme presupposes that all patients will have had their personality diagnoses determined by the IPDE. We envisage that this programme could (and should) be administered by any health professional with adequate understanding of personality disorder, the various categories and their characteristic traits. The health professional would not have to have had experience of using the IPDE him-/herself (because many health professionals would not) but he/she would need basic knowledge of the interview and how it scored.

What about settings in which the IPDE is not used? Although it would be preferable if the diagnosis of personality disorder is obtained with explicit criteria using a semi-structured approach, the programme still could be carried out if the diagnoses have been arrived at by other means, for instance, clinical opinion, but the clinician then would have to specify explicitly the traits that the individual possessed in order to justify a particular personality label. If this resulted in clinicians having to review the diagnostic 'label' of their patients and check if their patients do indeed fulfil the criteria for a specific personality diagnostic category, we see this as being a step forward rather than the reverse.

\section{Conclusion}

Readers of this article may well ask what all the fuss is about. Surely, the issue is simple - it is correct to give patients as much information as possible about their diagnosis to enable them to enter into a constructive therapeutic relationship with those who are providing therapeutic interventions. Our information - and we see no reason to doubt its generalisability - is that this diagnostic information for those with personality disorder is often not available and, even when it is (as in the case of the PDU), it is rarely transmitted to those who need it most - the patients. This has practical implications because the management of patients with personality disorder is generally regarded as difficult by psychiatrists; consequently, clinicians need all the help that they can get to increase their effectiveness. Transmitting information about the disorder is one way in which the therapeutic relationship may be strengthened and we suggest that by doing so can have a benefit for patient and psychiatrist alike.

\section{References}

AMERICAN PSYCHIATRIC

ASSOCIATION (1994) Diagnostic and

Statistical Manual of Mental Disorders (4th edn) (DSM-IV). Washington, DC: APA.
LEWIS, G. \& APPLEBY, L. (1988) Personality disorder: the patients psychiatrists dislike. British Journal of Psychiatry, 153, $44-49$ 
LORANGER, A.W., HIRSCHFELD,

R. M. A. SARTORIOUS, N. et al

(1991) TheWHO/ADAMHA

international pilot study of personality

disorders: background and purpose

Journal of Personality Disorders, $\mathbf{5}$

$296-306$

NHS EXECUTIVE (1997) The Patient's

Charter. Good Practice in Mental
Health Services; A Collection of

Good Practice in the Provision of

Community Mental Health Services.

London: NHS Executive.

STEPHENS, J. P. (2000) (letter) Is this

informed consent? Psychiatric Bulletin,

24,154 .
WORLD HEALTH ORGANIZATION (1992) ZIMMERMAN, M. (1994) Diagnosing

The ICD-10 Classification of Mental personality disorders. Archives of

and Behavioural Disorders. Geneva: General Psychiatry, 51, 225-245.

WHO.

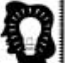

special

articles

*Karen D'Silva Specialist Registrar, Rampton Hospital, Retford, Nottinghamshire DN22 OPD Conor Duggan Professor of Forensic Mental Health, East Midlands Centre for Forensic Psychiatry 